\title{
Evaluation of Complement in Patients with Eosinophilic Pneumonia
}

\author{
Masayoshi Abe, Tomomochi GouyA*, Kiyoko TAnaKa, Teizo FuJItA**, Masayuki MryazaKI \\ and Motomichi Torisu*
}

\begin{abstract}
Complement evaluation was performed in two patients with active eosinophilic pneumonia and in one in remission, to determine the role of complement activation in the pathogenesis of this disorder. All three had cough, dyspnea, malaise, and blood eosinophilia; two patients also had pyrexia. In all 3 cases the pulmonary eosinophilic infiltrates (radiographic findings) and symptoms responded rapidly to steroid administration. The two patients with active eosinophilic pneumonia showed elevated CR3 but reduced FcrR on the PMN before and during steroid administration. In contrast PMN from four patients with bronchial asthma exhibited slightly elevated expression of both CR3 and FcrR during their asthma attack. It is suggested that clinical symptoms disappear soon after the beginning of steroid but changes of complement receptors on PMN may last for Ionger periods. On the basis of the combined results, this study indicates that estimation of complement activation may provide a useful indicator for disease activity in patients with eosinophilic pneumonia of unknown etiology.
\end{abstract}

(Internal Medicine 31: 717-724, 1992)

Key words: PIE syndrome, bronchial asthma, C3a, C4a, C5a, FcrR, CR3, DAF

\section{Introduction}

The diagnosis of pulmonary infiltrate with eosinophilia (PIE syndrome) is applied to those patients with pulmonary eosinophilia associated with marked blood eosinophilia (1). Because eosinophilic infiltration of the lung can exist in the absence of blood eosinophilia, it has been suggested that the term 'eosinophilic pneumonia', may be more appropriate than PIE syndrome (2). In the majority of patients, the etiology is unknown. The clinical course is chronic, characterized by persistent or recurrent pulmonary infiltrates and respiratory symptoms.

Although some investigators have suggested certain immunologic mechanisms for its occurrence and progression of this disorder (3), there has been little objective evidence in this respect. The complement-derived products, C3a and C5a, possess chemotactic activity for the neutrophils and eosinophils, and may play a role in their accumulation in the lung (4). Complement activation may perhaps stimulate the leukocytes to generate various arachidonate metabolites including leukotriene $\mathrm{B}_{4}$, a potent chemotactic factor for neutrophils and eosinophils $(5,6)$.
Few studies have systematically evaluated complement in patients with eosinophilic pneumonia over a period of time. We therefore investigated the role of complement in the development of this disorder using newer analytical methods in three patients, two with active disease who achieved remission on prednisolone and one during remission. Complement activation was evaluated by the complement split products in the fluid phase and by the receptors (CR3 and FcrR) and by complement regulating protein (DAF; decay-accelerating factor) expression on the cellular surface.

The results obtained suggest that the formation of immune complex and the activation of complement may stimulate the eosinophils and neutrophils in patients with eosinophilic pneumonia.

\section{Materials and Methods for Special Laboratory Examinations}

Blood was drawn using heparinized syringes from the patients or the healthy control (40-year-old man), without any drug administration for at least one year. PMN from this healthy man were always used as the contol for the receptor assay. After 3\% dextran sedimentation,

From the Research Institute for Diseases of the Chest and *the First Department of Surgery, Faculty of Medicine, Kyushu University, Fukuoka and **the Department of Immunology, Institute of Basic Medical Sciences, University of Tsukuba, Tsukuba

Received for publication October 19, 1991; Accepted for publication February 4, 1992

Reprint requests should be addressed to Dr. Masayoshi Abe, the Research Institute for Diseases of the Chest, Faculty of Medicine, Kyushu University, 3-1-1 Maidashi, Higashi-ku, Fukuoka 812, Japan 
polymorphonuclear leukocytes (PMN) and mononuclear cells were separated by centrifugation on FicollConray (specific gravity $=1.078$ ). Dextran-sedimented erythrocytes were washed twice by RPMI with $2 \%$ FCS (fetal calf serum). Eosinophils were separated in density gradients of Percoll (7). The erythrocytes, neutrophils, and eosinophils were suspended at a density of $1 \times 10^{7}$ cells $/ \mathrm{ml}$ of RPMI and reacted with fluorescein isothiocyanate-conjugated mouse anti-DAF (decayaccelerating factor) antibody, anti-Fc receptor for $\mathrm{IgG}$ (FcrR) monoclonal antibody (CD16; Becton-Dickinson, $\mathrm{CA}$ ), and phycoerythrin-conjugated anti-CR3 monoclonal antibody (CD11b; Becton-Dickinson, CA). After washing the cells with RPMI, the mean fluorescence intensity of 10,000 cells was analyzed with cytofluorography (FACscan, Ortho Diagnostics, MA) by adjusting the gain for each cell population. Values of the patients' $\mathrm{CR} 3, \mathrm{DAF}$, and FcrR were expressed as percentages of those of the healthy control.

$\mathrm{C} 3 \mathrm{a}, \mathrm{C} 4 \mathrm{a}$, and $\mathrm{C} 5 \mathrm{a}$ in plasma were assayed using RIA kits (Amersham, England). The normal ranges for $\mathrm{C} 3 \mathrm{a}$ and $\mathrm{C} 4 \mathrm{a}$ are $50-200 \mathrm{ng} / \mathrm{ml}$ and $50-250 \mathrm{ng} / \mathrm{ml}$, respectively. The level of $\mathrm{C} 5 \mathrm{a}$ in the patient plasma was always below the limit of detection $(10 \mathrm{ng} / \mathrm{ml})$.

\section{Case Histories}

\section{Case 1}

A 37-year-old man was admitted to Kyushu University Hospital with a 2-week history of a nonproductive cough, general malaise, and fever $\left(38.5^{\circ} \mathrm{C}\right)$. He was taking no medications. Pneumonia was diagnosed on chest X-ray.

Evaluation of his previous history revealed that he had experienced similar symptoms on two occasions: the previous summer and 7 years earlier. The more recent episode (1 year earlier) led to a diagnosis of suspected pulmonary tuberculosis for which the patient had received antituberculosis agents for 2 months. He discontinued taking the medications. This episode appeared therefore to be the third episode of unexplained pulmonary symptoms.

Physical examination on the first visit to our hospital revealed that he was not cyanotic. No rales were audible on auscultation. The blood pressure was $118 / 75 \mathrm{mmHg}$, heart rate was $70 / \mathrm{min}$ and respiratory rate was $15 / \mathrm{min}$. There was no adenopathy and the results of cardiac examination were normal. Examination of the abdomen revealed no abnormalities, and the extremities showed no clubbing or edema.

The peripheral white blood cell count was 19,600 and the red blood cell count was $448 \times 10^{4} / \mu$ l. The hematocrit was $44.0 \%$ and the hemoglobin was $15.0 \mathrm{~g} / \mathrm{dl}$. The platelet count was $475,000 / \mu \mathrm{l}$. The differential revealed $38 \%$ neutrophils, $22 \%$ lymphocytes, $1 \%$ monocytes and $39 \%$ eosinophils. No ova were found in the stool. Cold agglutinins were negative, as was antihepa-

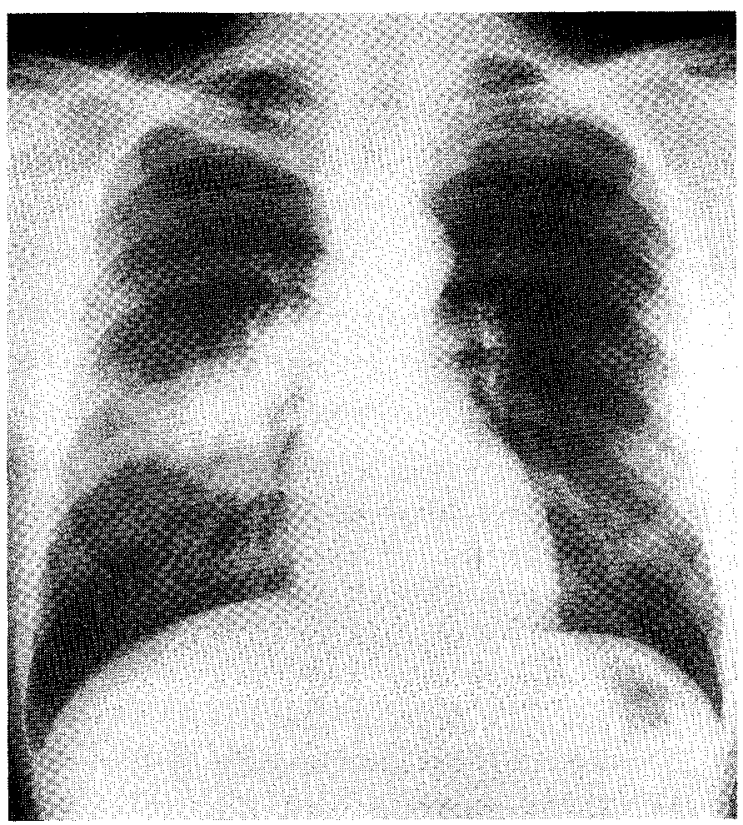

Fig. 1. Patient 1: Chest $X$-ray on the first visit.

titis B antibody. The chest X-ray on his first visit to our hospital is shown in Fig. 1. Routine serum chemistries showed a normal total protein, albumin, total bilirubin, direct bilirubin, GOT and GPT. The r-GPT was slightly elevated. Serum levels of IgG, IgA, IgM and IgE (RIST) were normal. Serum levels of total hemolytic complement activity, CH50 (56) and C3 (112 mg/dl) were both mildly elevated (normal range of $\mathrm{CH} 50$, $\mathrm{C} 3$, and $\mathrm{C} 4: 30-50,38-96 \mathrm{mg} / \mathrm{dl}$, and $11-46 \mathrm{mg} / \mathrm{dl}$, respectively). The time-course changes of these values are presented in Fig. 2,A. IgE (RAST) was positive for house dust, Dermatophagoides pteronyssinus and farinae, Aspergillus fumigatus and Candida albicans. Immunodiffusion studies for antibodies to Aspergillus, Candida and Cladosporium were all negative. The sputum showed numerous eosinophils. Gram stain of the sputum and sputum cultures were negative for acid-fast bacilli, fungi and bacterial pathogens.

Bronchoscopy (on day 2) revealed circumferential narrowing with a white mucous plug impacted in the right $\mathrm{B}^{6}$ orifice. The plug was removed with a forceps and examined histologically. It consisted of muco-sanguineous material with desquamated epithelial cells and eosinophils. No fungi were observed in this specimen upon staining with methenamine silver. Bronchial washings contained predominantly eosinophils and alveolar macrophages. A biopsy specimen of parenchymal tissue could not be obtained because the patient developed a severe attack of coughing during the procedure. The diagnosis was idiopathic eosinophilic pneumonia.

The administration of oral prednisolone ( $30 \mathrm{mg} /$ day) was begun and the patient became afebrile in one day. The shadow on the chest X-ray disappeared in two 


\section{Eosinophilic Pneumonia and Complement Activation}

(A)

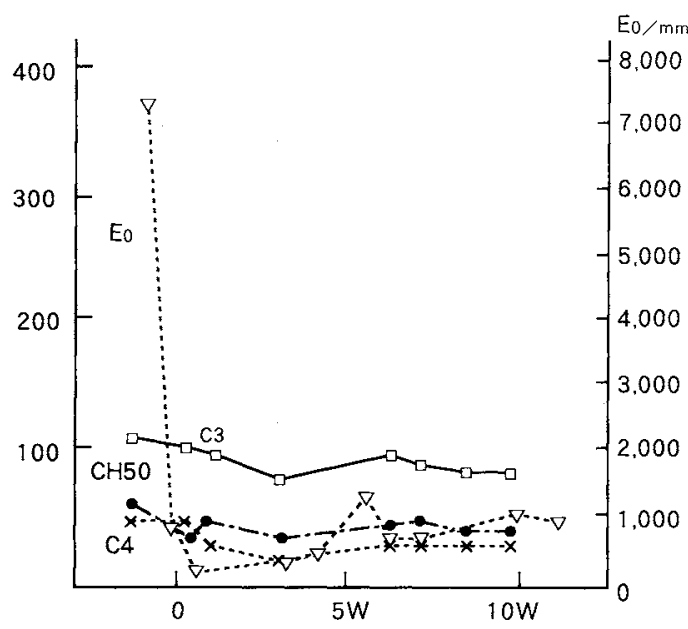

Prednisolone $\underbrace{4+1}_{30 \mathrm{mg}}$
(B)

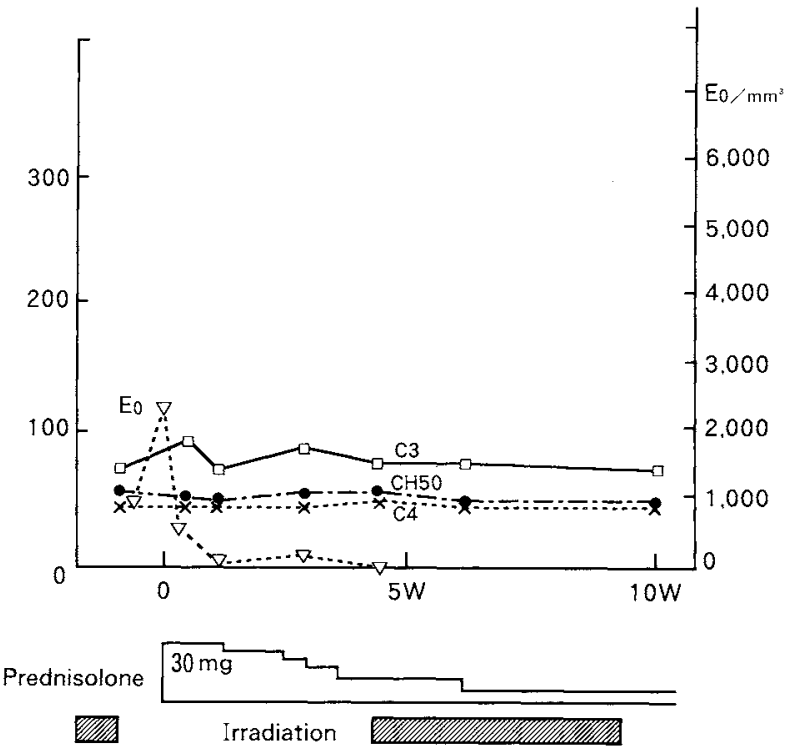

Fig. 2. Time-course studies for blood eosinophils, $\mathrm{E}_{0}(\nabla---\nabla), \mathrm{C} 3(\square-\square), \mathrm{C} 4(\times----\times)$, and CH50 $(\bullet-\cdot-)$ in patients 1 (A) and 2 (B). Blood eosinophil counts arc expressed as absolute numbers per microliter. Normal ranges of $\mathrm{C} 3, \mathrm{C} 4$, and $\mathrm{CH} 50$ are $38-96 \mathrm{mg} / \mathrm{dl}, 11-46 \mathrm{mg} / \mathrm{dl}$, and $30-50$. Abscissa indicates time (weeks) after initiation of prednisolone.

A) Oral prednisolone was initiated at $30 \mathrm{mg} /$ day and tapered off by $5 \mathrm{mg} / \mathrm{wk}$, and then discontinued after alternate day administration of $5 \mathrm{mg}$ for 6 days.

B) One week after the initiation of irradiation to the prostate $(2 \mathrm{~Gy} /$ day $\times 5)$ the patient developed a wheezing attack.

Oral prednisolone was started at a dose of $30 \mathrm{mg} /$ day and then tapered off.

Table 1. Data of Patients with Eosinophilic Pneumonia

\begin{tabular}{cccccc} 
Eosinophils & \multicolumn{3}{c}{ Complement $(n g / m l)$} & DAF expression & $C R 3$ \\
Patient & Lung Blood & C3a C4a C5a on PMN on Erythro. on PMN
\end{tabular}

1.

\begin{tabular}{|c|c|c|c|c|c|c|c|c|}
\hline \multicolumn{5}{|l|}{ During prednisolone } & $<10$ & $87 \%$ & & $114 \%$ \\
\hline $\begin{array}{c}1 \text { wk after the } \\
\text { initiation }\end{array}$ & + & - & 243 & 226 & $<10$ & $107 \%$ & & $135 \%$ \\
\hline 6 wks & - & - & 197 & 224 & $<10$ & $70 \%$ & $39 \%$ & $121 \%$ \\
\hline \multicolumn{9}{|l|}{ After prednisolone } \\
\hline $1 \mathrm{wk}$ & - & - & 299 & 171 & $<10$ & $75 \%$ & & $150 \%$ \\
\hline \multicolumn{9}{|l|}{ In remission } \\
\hline $5 \mathrm{mo}$ & - & - & 109 & 78 & $<10$ & $92 \%$ & $122 \%$ & $128 \%$ \\
\hline Before prednisolone & + & + & 297 & 972 & $<10$ & $99 \%$ & $50 \%$ & $112 \%$ \\
\hline \multicolumn{9}{|l|}{ During prednisolone } \\
\hline $1 \mathrm{wk}$ & + & - & 235 & 591 & $<10$ & $96 \%$ & & $105 \%$ \\
\hline $3 w k s$ & - & - & 3,600 & 1,370 & & & & \\
\hline $5 \mathrm{wks}$ & - & - & 341 & 1,520 & $<10$ & $137 \%$ & $49 \%$ & $261 \%$ \\
\hline $11 \mathrm{wks}$ & - & - & 125 & 599 & $<10$ & $104 \%$ & $100 \%$ & $80 \%$ \\
\hline \multirow{3}{*}{$\begin{array}{l}\text { Before predni } \\
\text { In remission } \\
1.5 \mathrm{y}\end{array}$} & + & + & $370^{*}$ & $156^{*}$ & $<10^{*}$ & & & \\
\hline & & & & & & & & \\
\hline & - & - & 136 & 114 & $<10$ & $103 \%$ & & $98 \%$ \\
\hline
\end{tabular}

* Plasma anaphylatoxin levels were assayed in a stored sample 
(A)

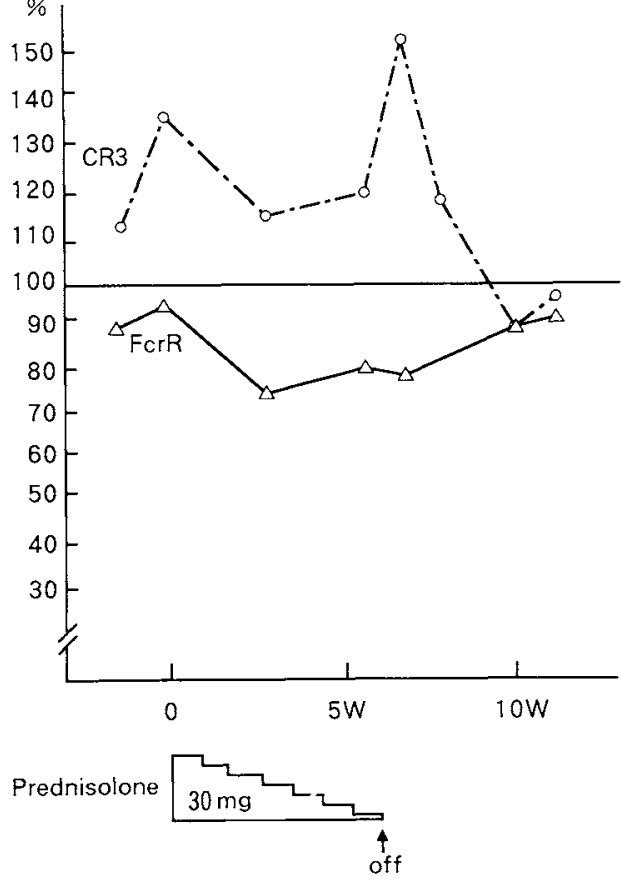

(B)

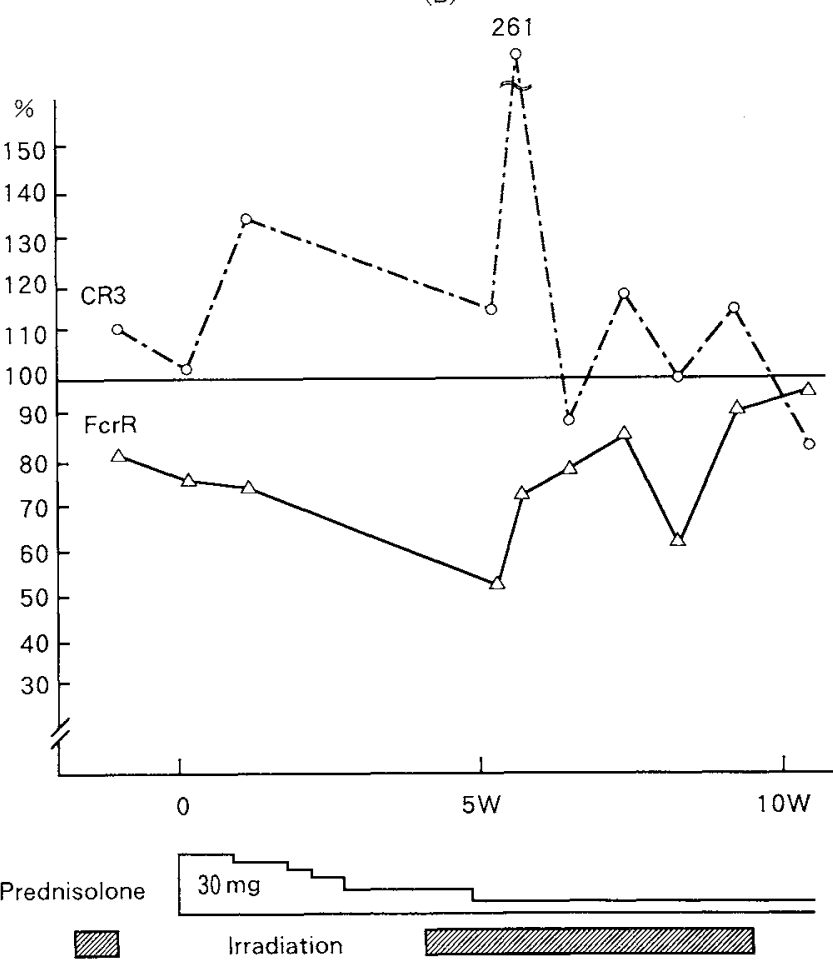

Fig. 3. Time-course studies for expressions of CR3 $\left(C_{-}-0\right)$ and $F \mathrm{crR}(\triangle-\triangle)$ on $\mathrm{PMN}$ from patients 1 (A) and 2 (B). Values of CR3 and FcrR are expressed as percentages of the normal control value. Others same as in Fig. 2.

weeks. The dosage of prednisolone was gradually tapered by $5 \mathrm{mg} /$ week. The patient's clinical course was uneventful and he remains well 6 months after the discontinuance of prednisolone. The time-course studies for special laboratory examinations are summarized in Fig. 3, A and in Table 1. As treatment with oral prednisolone resolved the pneumonia, the elevated $\mathrm{C} 3 \mathrm{a}$ was also gradually normalized over 2 weeks. CR3 and FcrR on neutrophils seemed to fluctuate as a mirror image. The patient's neutrophils, eosinophils, and erythrocytes showed a reduction in DAF expression on their surfaces; $70 \%, 45 \%$, and $40 \%$, respectively, as the lowest values examined. Following the cessation of prednisolone, CR3 on neutrophils and C3a in plasma were elevated transiently but both values spontaneously returned to normal levels in three weeks in association with DAF expression on neutrophils and erythrocytes.

\section{Case 2}

A 69-year-old man was admitted to the urology section for local irradiation to the urethral region following an operation for prostatic cancer. There was no evidence of metastatic disease to the lung, liver, bone or other organs according to chest X-ray and whole body CT. About one week after radiation therapy was begun the patient developed a nonproductive cough and an attack of wheezing. He was then referred to the pulmonary section for evaluation. Chest $\mathrm{X}$-ray revealed an infiltrative shadow in the right lower lung field (Fig. 4). He appeared pale but not cyanotic. Chest examination on his initial visit revealed fine crackling rales audible on the right lower chest. His blood pressure was $130 / 70 \mathrm{mmHg}$, heart rate 80 beats $/ \mathrm{min}$, and respiratory rate $18 / \mathrm{min}$. There

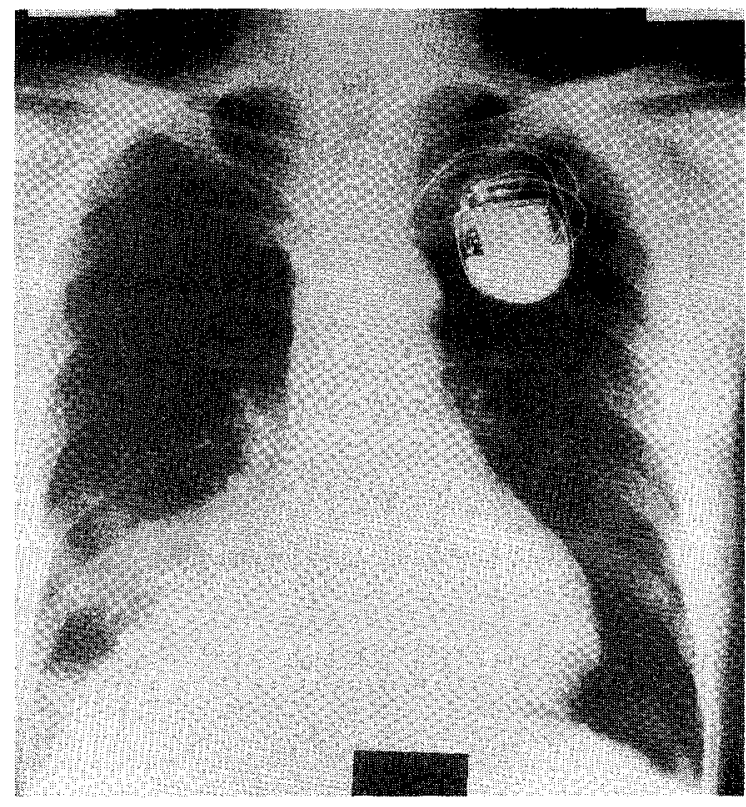

Fig. 4. Patient 2: Chest X-ray before initiating prednisolone. 
was no adenopathy. Two years earlier a pacemaker har been implanted for paroxysmal supraventricular tachy cardia (PSVT). The patient had taken a calcium channe antagonist and a beta-blocker for PSVT for more that 2 years. Cardiac examination showed normal ejection fraction. The pacemaker appeared to be functioning well. The abdomen showed no abnormalities and there was no clubbing or edema of the lower extremities.

The peripheral white blood cell count was 9,730 and the red blood cell count was $339 \times 10^{4} / \mu \mathrm{l}$. The hematocrit was $34.7 \%$ and the hemoglobin was $10.9 \mathrm{~g} / \mathrm{d} 1$. The platelet count was $27 \times 10^{4} / \mu \mathrm{l}$. The differential showed $39 \%$ neutrophils, $30 \%$ lymphocytes, $8 \%$ monocytes and 23\% eosinophils. Gram stain and acid-fast stain of the sputum were negative as were sputum cultures. Tests for cold agglutinins and anti-hepatitis B antibody were negative. Routine serum chemistry including total protein, albumin, GOT and GPT were all normal. Immunologic evaluation revealed an increased level of $\operatorname{IgG}(33.9 \mathrm{~g} / \mathrm{l})$ and $\operatorname{IgE}(2,700 \mathrm{IU} / \mathrm{ml})$. No ova were found in the stool. Serum levels of total hemolytic complement activity, C3, and $\mathrm{C} 4$ were all within normal limits, as listed in Fig. 2,B. IgE (RAST) was positive for Candida albicans but negative for Aspergillus, house dust, and Dermatophagoides pteronyssinus. The infiltrative shadow on the chest X-ray was enlarged concomitantly with the exacerbation of his dyspnea.

Bronchoscopy was performed and circumferential narrowing and impaction by a white mucous plug was found in the right middle bronchus. Histological examination of a lung biopsy specimen revealed mild fibrosis and fibrin deposition in the interstitium with moderate infiltration by eosinophils and macrophages (Fig. 5). Staining with methenamine silver was negative for fungi.

Oral prednisolone $(30 \mathrm{mg} /$ day) was started, leading to relief of dyspnea in one day. The infiltrative shadow disappeared within a few weeks of prednisolone administration. Time-course studies for special laboratory examination for this patient are summarized in Fig. 3,B and Table 1 . The eosinophilic pneumonia began 1 week after the beginning of irradiation to the prostate. Initiation of prednisolone and interruption of radiotherapy rapidly improved the patient's symptoms and chest $\mathrm{X}$-rays but the resumption of irradiation was followed by an increase of CR3 on PMN. FcrR and CR3 on neutrophils also seemed to fluctuate as a mirror image. These results suggest that the local irradiation may have facilitated the occurrence and progression of eosinophilic pneumonia in this patient.

\section{Case 3}

A 50-year-old woman came to the hospital with a productive cough, general malaise, and fever $\left(38.0^{\circ} \mathrm{C}\right)$. She had no history of drug allergy. The serum IgE level was normal. Total white cell count was $11,200 / \mu 1$ and the erythrocyte sedimentation rate was $106 \mathrm{~mm} / \mathrm{h}$. CH50

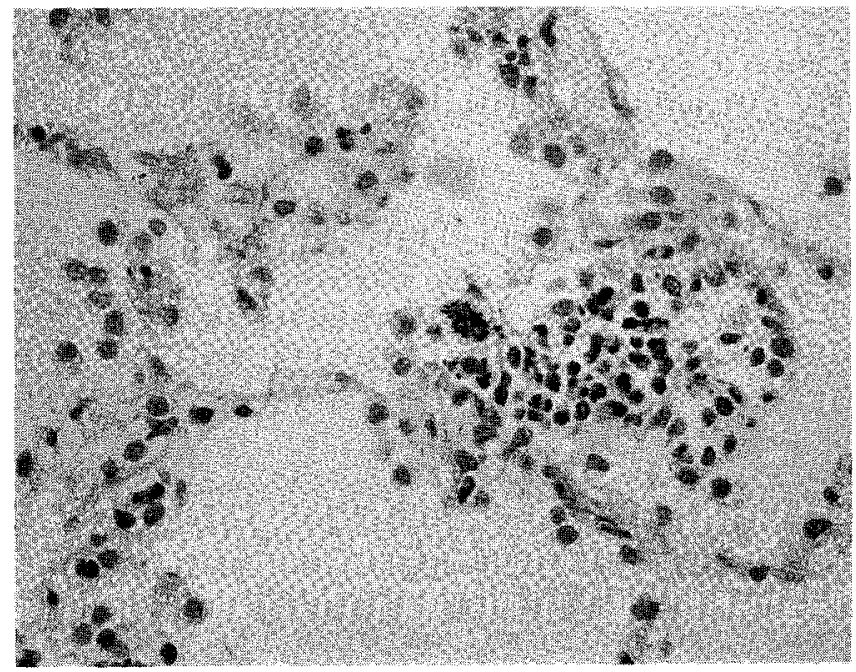

Fig. 5. Patient 2: Transbronchial lung biopsy shows mild fibrin deposition in the interstitium with moderate eosinophil and macrophage infiltration. H-E, magnification $\times 200$.

(=60) and C3 $(=98 \mathrm{mg} / \mathrm{dl})$ were both mildly elevated. Chest X-ray revealed infiltrative shadows in both upper fields (Fig. 6). The intravenous administration of antibiotics, including minomycin and cephalosporin for 5 days, failed to improve the symptoms. Two weeks later, the peripheral white cell count was 11,100 and the red blood cell count was $375 \times 10^{4} / \mu 1$. The hematocrit was $35 \%$ and the hemoglobin was $11.3 \mathrm{~g} / \mathrm{dl}$. The plate-

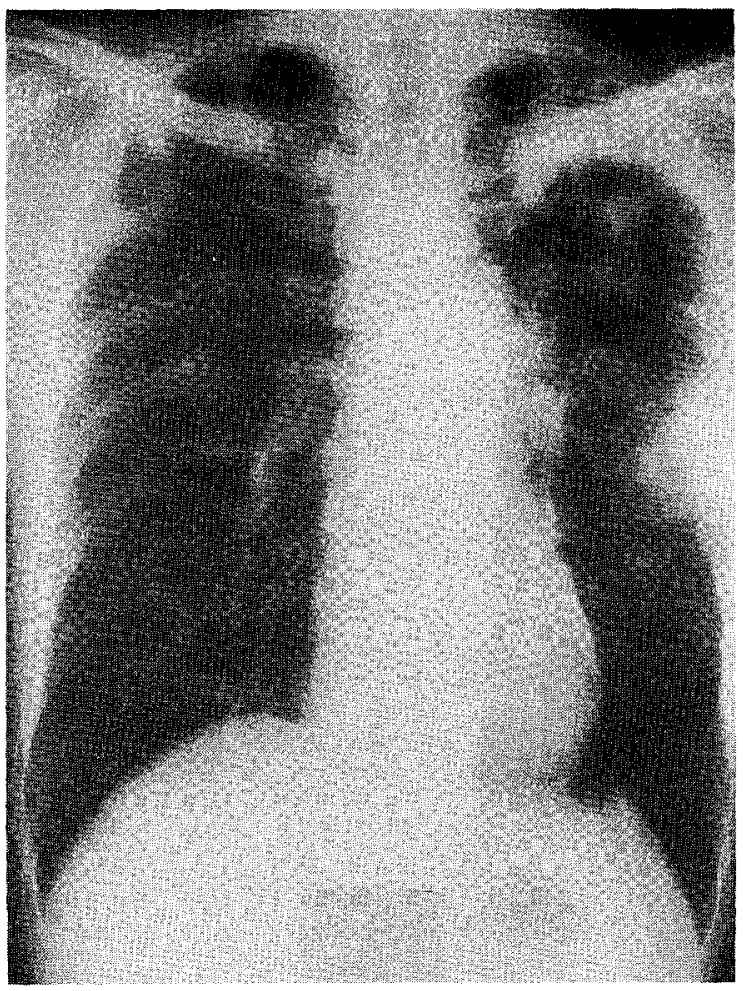

Fig. 6. Patient 3: Plain chest roentgenogram before the initiation of prednisolone. 


\section{Abe et al}

let count was $38.6 \times 10^{4} / \mu$ l. The differential showed $46 \%$ neutrophils, $29 \%$ lymphocytes, $8 \%$ monocytes and $17 \%$ eosinophils. Sputum cytology revealed numerous eosinophils.

Eosinophilic pneumonia was strongly suspected. Accordingly, oral prednisolone was started at a dose of $30 \mathrm{mg} /$ day. The temperature returned to normal within one day; the chest X-ray was normal two weeks later except for a small area of scarring at the right apex which predated the present illness. Prednisolone was gradually withdrawn over the next six months. The patient has remained clinically and radiologically well for one and one half years. During her remission, we evaluated C3a, $\mathrm{C} 4 \mathrm{a}$, and C5a in plasma and the expression of DAF, CR3, and FcrR on PMN. Plasma levels of C3a, C4a, and C5a were 136, 114, and less than $10 \mathrm{ng} / \mathrm{ml}$, respectively. Expressions of DAF, CR3, and FcrR on PMN were 103,
98 , and $95 \%$ of the normal control, respectively (Table 1 ). We could evaluate anaphylatoxin levels only before prednisolone administration by using the plasma stored at $-20^{\circ} \mathrm{C}$, which showed mildly elevated C3a (see Table 1).

\section{$D A F$ expressions on erythrocytes}

In cases 1 and 2, DAF expression on erythrocytes was reduced to approximately one-half of the value obtained in a normal control (Fig. 7 and Table 1). In case 1, hemoglobin was reduced to $13.3 \mathrm{~g} / \mathrm{dl}$ from $15.0 \mathrm{~g} / \mathrm{dl}$ during the acute illness and in case 2, to $7.9 \mathrm{~g} / \mathrm{dl}$ from $10.9 \mathrm{~g} / \mathrm{dl}$ with no evidence of GI bleeding, suggesting a presumptive correlation with reduced DAF expression.

Complement evaluation in patients with bronchial asthma

Complement was similarly evaluated during an asthma attack in four patients with bronchial asthma (two intrin-
(A)
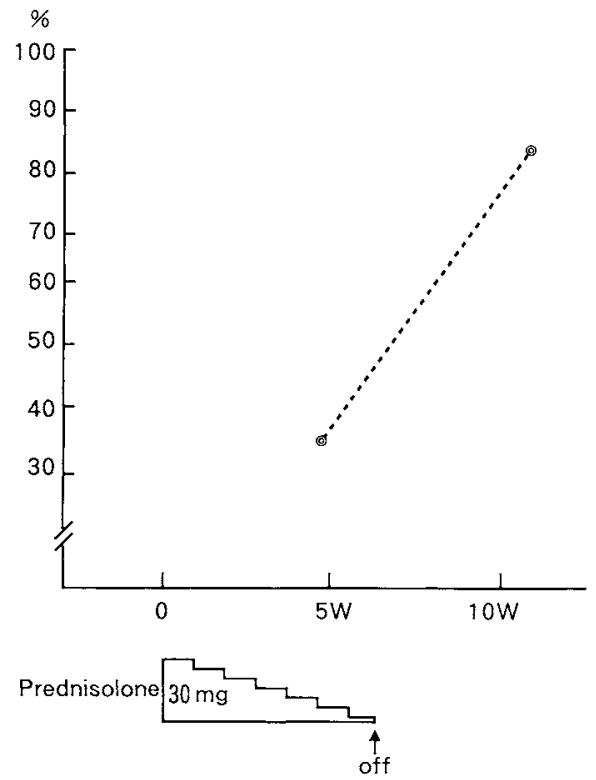

(B)

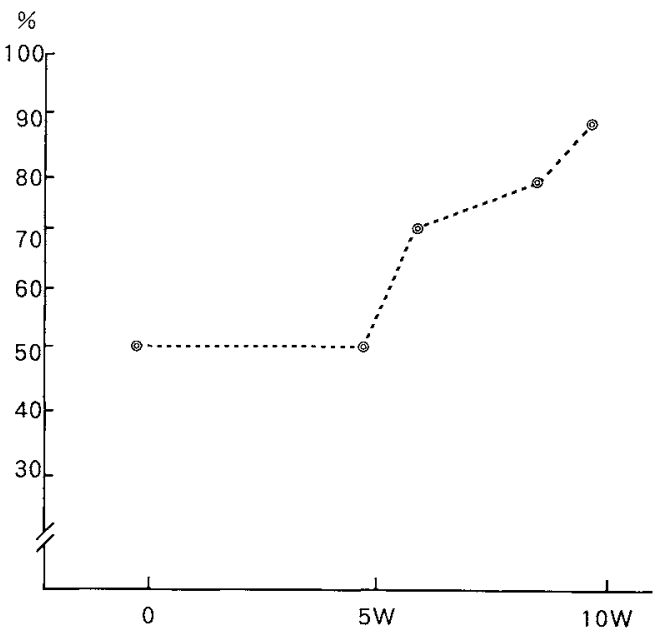

Prednisolone

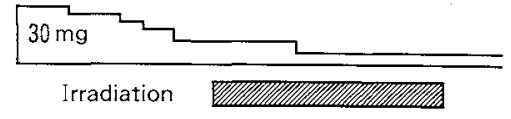

Fig. 7. Time-course studies for expressions of DAF on erythrocytes from patient 1 (A) and 2 (B). Others same as in Fig. 2.

Table 2. Data Summary of Patients with Bronchial Asthma during an Attack

\begin{tabular}{|c|c|c|c|c|c|c|c|c|c|}
\hline & & $\begin{array}{c}\mathrm{C} 3 \\
(38-96)^{*}\end{array}$ & $\begin{array}{c}\mathrm{C} 4 \\
(11-46)\end{array}$ & $\begin{array}{c}\text { CH50 } \\
(30-50)\end{array}$ & $\begin{array}{c}C 3 a \\
(50-200)\end{array}$ & $\begin{array}{c}\mathrm{C} 4 \mathrm{a} \\
(50-250)\end{array}$ & CR3 & $\mathrm{FcrR}$ & DAF \\
\hline \multirow{2}{*}{ Ex } & Patient 1 & 95 & 29 & 45 & 249 & 103 & $110 \%$ & $116 \%$ & $126 \%$ \\
\hline & Patient 2 & 107 & 45 & 48 & 293 & 306 & $108 \%$ & $108 \%$ & $79 \%$ \\
\hline \multirow{2}{*}{ In } & Patient 3 & 78 & 31 & 44 & 225 & 152 & $117 \%$ & $153 \%$ & $100 \%$ \\
\hline & Patient 4 & 71 & 27 & 41 & 176 & 60 & $95 \%$ & $104 \%$ & $100 \%$ \\
\hline
\end{tabular}

While patients 1 (18y.o., male) and 2 (66y.o., female) suffered from the extrinsic typc (Ex) of bronchial asthma, patients 3 (73 y.o., female) and 4 (58y.o., female) suffered from the intrinsic type (In). All four patients were free of systemic administration of corticosteroid. Asthma attack was diagnosed by complaint of dyspnea attack and reduction of arterial $\mathrm{PO}_{2}$ at more than $10 \mathrm{mmHg}$. *Normal ranges are shown in parentheses 
sic and two extrinsic types). Chest X-ray examination of these patients revealed no pneumonic shadow. These four patients were treated with xanthine derivatives and beta-agonists, but not with systemic administration of steroid hormones. As shown in Table 2, one patient (case 2) showed an increased $\mathrm{C} 3$ and three of four patients had mildly elevated C3a values. These four patients showed normal or elevated CR3 and FcrR expressions on PMN, in contrast to the two patients with active eosinophilic pneumonia.

\section{Discussion}

In these three patients, eosinophilic pneumonia was suspected because of the presence of pulmonary infiltrative shadows, marked blood and lung eosinophilia and the rapid response to steroid administration (8). Immunologic abnormalities have been the subject of speculation in idiopathic eosinophilic pneumonia because the prominence of eosinophils in the lung and the peripheral blood suggests a contribution of the antigenantibody reaction to the pathologic process. Since the antigen-antibody complex may occupy FcrR (CD 16) and reduce the receptor numbers, the reduced FcrR expression on PMN during the active stage in the first two patients may indicate prior activation with antigenantibody complex (9). It cannot be denied, however, that such a reduced FcrR may be due to steroid effects (10).

Fox and Seed (11) described three cases of chronic eosinophilic pneumonia all of whom had normal serum IgE levels (similar to patients 1 and 3 ). They speculated that the activation of complement appeared to be the stimulus for eosinophilia in type 2 (cytotoxic) and 3 (immune complex) reactions. However, they found little evidence of complement activation in their patients, because they used only the classical methods, CH50, $\mathrm{C} 3$ and C4. In the first two patients of the present study, the elevation of $\mathrm{C} 3 \mathrm{a}$ and/or C4a in plasma was detected before the administration of prednisolone. C3a gradually normalized after the initiation of prednisolone. A mild (in patient 1) or marked elevation of plasma C3a (in patient 2) was found immediately after the cessation or rapid withdrawal of prednisolone, suggesting a steroid withdrawal effect. The higher C4a elevation in patient 2 suggests complement activation preferentially via the classical pathway in this patient. Additionally, the elevated CR3 expression on PMN during the active stage may also support the complement activation on the cellular surface. These results suggest that estimation of complement activation may provide a useful indicator of disease activity in eosinophilic pneumonia.

A complement regulating protein, the decay-accelerating factor (DAF) residing on PMN from the patients' blood, was examined by monoclonal antibody against this protein and by fluorescence-activated flow cytometry (FACS). DAF is a glycoprotein of approximately $70 \mathrm{KD}$ which down-regulates $\mathrm{C} 3$ and C5 convertase (12-14). In patient 1, the value of DAF molecules on the patient's neutrophils, eosinophils and erythrocytes was less than the normal control value before and during steroid treatment and immediately after its cessation. In contrast, DAF expression on PMN from case 2 was increased during steroid treatment. The differences in the elevated anaphylatoxin subclass and DAF expression on PMN may indicate different facilitating factors between the two patients, especially the effect of local irradiation in case 2 .

It has been established that patients with paroxysmal nocturnal hemoglobinuria (PNH) lack DAF expression on their erythrocytes $(15,16)$ and its deficiencies are causally related to hemolysis. Anemia in some patients with eosinophilic pneumonia has been reported by Fox and Seed (11). Therefore, DAF expression on the patients' erythrocytes was examined and found to be only $39 \%$ of the normal control value in patient 1 and $50 \%$ in patient 2 . In these two patients, a transient decrease in hemoglobin with unknown etiology was noted and these results proposed a correlation of anemia with the decreased DAF expression. Therefore, it cannot be excluded that transient anemia may be related to a reduced DAF expression on the erythrocytes and consequently autologous complement-mediated hemolysis.

In the first 2 patients the elevated plasma $\mathrm{C} 3 \mathrm{a}$, increased CR3 and decreased FcrR on PMN as well as the decreased DAF expression on the erythrocytes all returned to normal (with a delay) after the remission of the objective signs (blood eosinophilia and chest X-ray) and symptoms. The delayed restoration of increased complement fragments and altered receptor expression may explain a frequent relapse after a rapid withdrawal of steroid. In the third patient examined during remission, the plasma $\mathrm{C} 3 \mathrm{a}, \mathrm{C} 4 \mathrm{a}$, and $\mathrm{C} 5 \mathrm{a}$ were within normal limits as were the expressions of CR3, DAF, and FcrR on PMN.

Regarding the comparison of complement activation in eosinophilic pneumonia with that in other allergic disorders, these parameters were examined in four patients with bronchial asthma. Since these complement parameters were within normal limits during remission we evaluated these parameters during their attack. The PMN from these asthma patients showed rather increased expressions of FcrR in contrast to the patients with eosinophilic pneumonia.

In conclusion, these observations may implicate the production of antigen-antibody complex and the subsequent activation of complement in the development and progression of eosinophilic pneumonia. The present results may warrant further evaluation of complement activation in patients with idiopathic eosinophilic pneumonia. 
Acknowledgments: We thank Drs. Hiroshi Hasuo, Toru Fukai, Tetsuo Yasumasu, and Arahito Mitsutake for their support of this study. This study has been supported in part by a Grant-in-Aid from the Ministry of Education, Science and Culture of Japan.

\section{References}

1) Reeder $\mathrm{WH}$, Goodrich BE. Pulmonary infiltration with eosinophilia (PIE syndrome). Ann Int Med 36: 1217, 1952.

2) Liebow AA, Carrington $\mathrm{CB}$. The eosinophilic pneumonias. Medicine 48: 251, 1969.

3) Colby TV, Carrington CB. Infiltrative Lung Disease, Pathology of the Lung, Thurlbeck WM, Ed. Thieme Medical Publishers, Inc., New York, 1988, p. 425.

4) Kay AB, Shin HS, Austen KF. Selective attraction of eosinophils and synergism between eosinophil chemotactic factor of anaphylaxis (ECF-A) and a fragment cleaved from the fifth component of complement (C5a). Immunology 24: 969, 1973.

5) Hugli TE. Structure and function of the anaphylatoxins. Springer Semin Immunopathol 7: 193, 1984.

6) Hugli TE, Muller-Eberhard HJ. Anaphylatoxins: C3a and C5a. Adv Immunol 26: 1, 1978.

7) Gartner I. Separation of human eosinophils in density gradients of polyvinylpyrrolidone-coated silica gel (Percoll). Immunology 40: 133, 1980.

8) Fraser RG, Pare JAP, Pare PD, et al. Eosinophilic lung disease. in: Diagnosis of Diseases of the Chest. 3rd ed., WB Saunders, Philadelphia, 1989, p. 1290.
9) Boros P, Gardos E, Bekesi GJ, et al. Change in expression of FcrRIII (CD16) on neutrophils from human immunodeficiency virus-infected individuals. Clin Immunol Immunopathol 54: 281, 1990.

10) Ruiz P, Gomez F, King M, et al. In vivo glucocorticoid modulation of guinea pig splenic macrophage Fcr receptors. J Clin Invest 88: 149, 1991.

11) Fox B, Seed WA. Chronic eosinophilic pneumonia. Thorax 35: $570,1980$.

12) Berger $M$, Medof ME. Increased expression of complement decay-accelerating factor during activation of human neutrophils. J Clin Invest 79: 214, 1987.

13) Nicholson-Weller A, Spicer DB, Austen KF. Deficiency of the complement regulatory protein, "decay-accelerating factor," on membranes of granulocytes, monocytes, and platelets in paroxysmal nocturnal hemoglobinuria. N Engl J Med 312: 1091, 1985

14) Fujita $T$, Inoue $T$, Ogawa $K$, et al. The mechanism of action of decay-accelerating factor (DAF); DAF inhibits the assembly of $\mathrm{C} 3$ convertases by dissociating $\mathrm{C} 2 \mathrm{a}$ and $\mathrm{Bb}$. J Exp Med 166: $1221,1987$.

15) Nicholson-Weller A, March JP, Rosenfeld SI, et al. Affected erythrocytes of patients with paroxysmal nocturnal hemoglobinuria are deficient in the complement regulatory protein, decay accelerating factor. Proc Natl Acad Sci USA 80: 5066, 1983.

16) Pangburn MK, Schreiber RD, Muller-Eberhard HJ, et al. Deficiency of an erythrocyte membrane protein with complement regulatory activity in paroxysmal nocturnal hemoglobinuria. Proc Natl Acad Sci USA 80: 5430, 1983. 